\title{
LA SATISFACCIÓN CON LA FAMILIA Y SU RELACIÓN CON LA AGRESIVIDAD Y LA INTELIGENCIA EMOCIONAL EN ADOLESCENTES
}

\author{
Natalia Larraz Rábanos, Eva Urbon Ladrero, José Luis Antoñanzas y Carlos Salavera \\ Universidad de Zaragoza, España
}

\section{Resumen}

La inteligencia emocional (IE) ha sido ampliamente estudiada en las últimas décadas. Desde los primeros trabajos hasta nuestros días, son muchas y variadas las aportaciones que se han realizado por parte de la comunidad científica sobre este constructo. Pero también hay que señalar que las investigaciones sobre la relación de la IE con variables como la agresividad y la satisfacción con la familia en jóvenes no son tan frecuentes. Los adolescentes en su devenir hacia la edad adulta se enfrentan a toda una serie de dificultades. La agresividad constituye una de ellas. La violencia entre los mas jóvenes ha ido aumentando sistemáticamente en las aulas y fuera de ellas. En este sentido, la familia como agente socializador básico y la Inteligencia Emocional como habilidad fundamental para el autocontrol del sujeto parecen necesarias. Se realizó un estudio en dos centros educativos de Secundaria para comprobar la relación de la satisfacción con la familia con la agresividad y con la IE. Los resultados pusieron de manifiesto dicha relación. La satisfacción con la familia es fundamental para un mejor control de la ira y un buen uso de las habilidades emocionales.

Palabras clave: Satisfacción familiar, Inteligencia emocional, Agresividad, Adolescentes.

\section{Abstract}

Emotional intelligence (EI) has been extensively studied in recent decades. From the first works to the present day, there are many and varied contributions that have been made by the scientific community on this construct. But it should also be noted that research on the relationship of EI with variables such as aggressiveness and satisfaction with the family in young people is not so frequent. Adolescents as they move into adulthood face a whole series of difficulties. Aggression is one of them. Violence among the youngest has been systematically increasing in classrooms and outside of them. In this sense, the family as a basic socializing agent and Emotional Intelligence as a fundamental ability for the subject's self-control seem necessary. A study was carried out in two secondary schools to verify the relationship of satisfaction with the family with aggressiveness and with EI. The results revealed this relationship. Satisfaction with family is essential for better anger management and good use of emotional skills.

Keywords: Family satisfaction, Emotional intelligence, Aggression, Adolescents.

Correspondencia.

\section{Natalia Larraz Rábanos nlarraz@unizar.es}

Departamento de Psicología y Sociología (Grupo de investigación Observatorio para la Investigación e Innovación en Ciencias Sociales. OPIICS).

Facultad de Educación. Universidad de Zaragoza

Pedro Cerbuna, 12. 50009, Zaragoza. 


\section{Introducción}

Durante los últimos años el estudio de la inteligencia emocional ha suscitado un gran interés. Haciendo un breve recorrido histórico de su conceptualización, las aportaciones de Gardner (1983) y Sternberg (1986), incluyen en sus teorías conceptos como la capacidad de adaptación al contexto y las competencias interpersonales e intrapersonales. Goleman (1996) populariza el término y desglosa la inteligencia emocional en cuatro competencias: autoconciencia emocional, auto-motivación, empatía y habilidades sociales. Mayer y Salovey (1997) la definen como la habilidad de percibir, expresar, comprender y regular las propias emociones y las de los demás, promoviendo un crecimiento emocional e intelectual. Por último, otro autor destacado en dicho constructo es Bar-On (1997) que entiende la inteligencia emocional como un conjunto de competencias no cognitivas que permiten afrontar las dificultades de forma exitosa.

Las personas emocionalmente inteligentes además de tener mayor capacidad para percibir, comprender y regular las propias emociones (repercutiendo esto de forma positiva en su bienestar personal), son capaces de generalizar estas habilidades a las emociones de los demás haciendo, de este modo, sus relaciones sociales, familiares y personales más saludables. Las personas con un déficit en inteligencia emocional presentan mayor predisposición a tener estrés, ansiedad, depresión y problemas interpersonales; en cambio, un alto coeficiente emocional se asocia con una buena salud física y mental (Ciarrochi, Chan y Caputi, 2000; Extremera y Fernández-Berrocal, 2005; Ferragut y Fierro, 2012).

\section{Agresividad e inteligencia emocional}

La palabra agresividad, proveniente de agresivo, formada con raíces latinas "aggredi" y significa "relativo a hacer daño a alguien". Ha sido definida por la RAE (2020) como la tendencia a actuar o responder violentamente o acometividad (propensión a acometer, atacar o embestir; o brío, pujanza, decisión para emprender algo y arrostrar sus dificultades). Es decir, la agresividad estaría asociada a la destrucción o violencia, ya sea hacia uno mismo o hacia los demás (Contini, 2015). En este caso, se hace referencia a que la agresividad, estaría intrínsecamente relacionada con la ira, descrita como una de las seis emociones básicas propuestas por Damásio (2005) además del miedo, el asco, la sorpresa, la tristeza y la alegría. Dichas emociones estarían basadas en el principio de placer/displacer o placer/dolor que tienen como base las pulsiones. La pulsión fue definida en primera instancia por Freud (1976) como la energía somato-psíquica (aspecto situado entre lo corporal o somático y lo mental o psíquico) que hace que el organismo tienda a la consecución de un fin (Laplanche y Pontalis, 2004). Es decir, la ira sería un componente emocional eminentemente psíquico que se activa y moviliza al organismo hacia un fin, que sería calmar dicho estado interno (causado por sentimientos, pensamientos, ideaciones o fantasías) o externo (causado por personas u objetos).

La relación existente entre la agresividad y las habilidades sociales ocurre que mientras las habilidades sociales constituyen la posibilidad de interactuar con los otros, pares y adultos, de una manera mutuamente satisfactoria, las dificultades en ellas, obstaculizan el vínculo y la relación con los otros, principalmente a través del retraimiento y el aislamiento social por un lado y de la agresividad, por el otro (Contini, et al. 2012).

La adolescencia es una etapa del crecimiento en la que se producen grandes cambios en la personalidad, al mismo tiempo que se define la identidad sexual y personal independiente de los padres y se elabora un proyecto de vida, en el cual comienzan o se incrementan los problemas externalizantes (conducta antisocial, agresividad, violencia) e internalizantes (timidez, ansiedad social) como un fenómeno complejo y multicausal (Contini, et al. 2012).

Uno de los estudios más significativos relacionada con la agresividad y sus efectos en las habilidades sociales ha sido el realizado por Kohn (1977), en el que analiza investigaciones efectuadas entre 1920 y 1960, y le permite enunciar la teoría bifactorial que afirma la existencia de dos factores definidos en la agresividad: retraimiento y agresividadconducta antisocial. Por otro lado, un modelo contemporáneo interesante para analizar las habilidades sociales y sus déficits es el Modelo del Aspa (Silva, Martínez y Generós, 1997) en el que se proponen dos grandes dimensiones bipolares: a) conducta prosocial-conducta antisocial y b) alta sociabilidad-baja sociabilidad. En las investigaciones con dicho modelo, identificaron que la baja sociabilidad se asociaba a ansiedad social, timidez y retraimiento, mientras que el comportamiento antisocial aparecía vinculado a agresividad, obstinación, oposición a las normas y a comportamientos delictivos. Por lo que estos resultados son acordes a lo observado según la literatura científica, en la que se indica que el desarrollo de la empatía, integra procesos cognitivos y emocionales implicados en la disposición prosocial que contribuyen a una conducta prosocial más autónoma y madura como contrapuesta a la conducta agresiva (Mestre, Samper, Tur-Porcar et al., 2012).

De este modo, se podría afirmar la existencia de una relación entre conducta prosocial y una disminución de las conductas agresivas, del mismo modo que la conducta prosocial supondría una elevada inteligencia emocional (IE en adelante) y un desarrollo elevado de las habilidades sociales.

Según se ha observado en la literatura, son escasos los estudios que analizan la relación entre conducta agresiva e IE, aunque la mayoría de ellos trata de probar una relación 
significativa entre ambos constructos, mostrando que las altas puntuaciones en conductas agresivas presentan un bajo nivel de IE y viceversa (Inglés et al., 2014). En este sentido, los resultados de un estudio de Bohnert, Crnic y Lim (2003) revelaron que los niños que presentaban más conductas agresivas, además de presentar diferencias en la expresión y modulación de la ira, tenían mayor dificultad para comprender las emociones y las causas de las mismas.

Inglés et al. (2014) en un estudio llevado a cabo, examinan la relación entre IE rasgo y los componentes: motor (agresividad física $\mathrm{y}$ agresividad verbal), cognitivo (hostilidad) y afectivo/emocional (ira) de la conducta agresiva en la población adolescente de 12 a 17 años de edad ( $N$ =314). Los resultados indicaron que los adolescentes con altas puntuaciones en conducta agresiva física, verbal, hostilidad e ira, presentaron puntuaciones significativamente más bajas en IE rasgo que sus iguales con puntuaciones bajas en conducta agresiva física, verbal, hostilidad e ira. Del mismo modo, en otros estudios han señalado la impulsividad como la principal predictora de la conducta agresiva y los mecanismos de control cognitivo caracterizados por la rumiación y la preocupación como variables predictoras de la agresión verbal y el resentimiento (López Del Pino, Sánchez, Pérez et al., 2008).

Otro estudio con adolescentes de entre 14 y 19 años, demostró una asociación existente entre una persona alta en IE y menores conductas agresivas, concluyendo que aquellos con una tendencia baja en justificar sus comportamientos agresivos, mostraban una mayor claridad emocional, mayor facilidad para solucionar sus emociones negativas, mantener en el tiempo las emociones positivas y, por tanto, desarrollar una mejor salud mental (Extremera y Fernández -Berrocal, 2002). Asimismo, los adolescentes que son emocionalmente inteligentes detectan mejor las presiones emocionales por parte de sus compañeros, siendo más capaces de afrontar las diferencias entre sus propias emociones y las del resto de compañeros, al contrario de lo que ocurre con adolescentes que tienen menor control emocional (Trinidad y Johnson, 2002). En este sentido, Garaigordobil y Oñederra (2010), estudiaron la relación entre acoso escolar y determinadas características asociadas a la inteligencia emocional (emotividad, eficacia, pensamiento supersticioso, rigidez, pensamiento esotérico e ilusión) en una muestra de 248 estudiantes de 12 a 16 años. Los resultados muestran que tanto los adolescentes que habían sido víctimas de bullying como los adolescentes acosadores, presentaron un bajo nivel de inteligencia emocional.

Siu (2009) muestra en su investigación, una relación inversa entre inteligencia emocional y problemas de conducta. Además, los resultados de este estudio pusieron de manifiesto que la autogestión de las emociones se relacionó negativamente con todo tipo de problemas de conducta, y que el manejo inadecuado de las emociones puede conducir a niveles altos de problemas de conducta, tales como agresividad y delincuencia.

Por último, según Diosa, Rodríguez y Romero (2013), existe una asociación entre la gestión inadecuada de las emociones y la aparición de conducta agresiva; concretamente la agresividad está asociada con un déficit de la comprensión y de la regulación emocional.

\section{Agresividad, inteligencia emocional y satisfacción fami- liar}

En una revisión de las distintas teorías psicológicas sobre la agresión, Carrasco y González (2006) mencionan que ya desde incluso antes de los años 70 se han ido desarrollando numerosas teorías que relacionan la conducta agresiva en la infancia con los hábitos de crianza de los padres, contemplando el rechazo, la falta de apoyo y afecto, el uso del castigo para controlar la conducta del niño, la falta de supervisión y de comunicación y una disciplina inconsistente, como las variables más significativas en relación a la conducta agresiva.

En estudios realizados con adolescentes y el estilo parental, la percepción de un estilo parental autoritario, parecería asociado al incremento de las manifestaciones de agresividad, dado que la percepción de un nivel bajo de afecto y un elevado grado de control, propio de este estilo, podría deteriorar seriamente el ajuste que los adolescentes muestran en diferentes contextos como el de las interacciones con los iguales (De la Torre-Cruz, García-Linares y CasanovaArias, 2014). Del mismo modo, un estilo parental democrático se relaciona con un mejor ajuste psicosocial en niños y adolescentes debido a un mayor afecto y aceptación que hace que se muestren más receptivos a las influencias paternas. Además de la adecuada combinación de implicación e imposición de límites que facilita el desarrollo de habilidades de autorregulación y les permite actuar de manera competente. Por último, la promoción del intercambio verbal entre padres e hijos hace a éstos últimos partícipes de un proceso que fomenta la competencia cognitiva y social, incrementando su nivel de actuación más allá del marco familiar (Steinberg, 2001) y fomentando una percepción en el adolescente de unas influencias parentales positivas (afecto y calidad de cuidados), lo que sirve como factor protector y reduce la posibilidad de manifestaciones violentas y antisociales. Oliva (2006) realiza un análisis pormenorizado de las relaciones familiares y el desarrollo adolescente en relación a los hallazgos más significativos que se han realizado en las últimas décadas sobre el tema y pone atención especial sobre dos aspectos: los cambios en las relaciones con la llegada de la adolescencia, su influencia en el desarrollo y el ajuste del adolescente. Además, indica que las claves para un adecuado estilo parental son el afecto, la comunicación, la adecuada gestión de conflictos y el control y la promoción de la autonomía en el adolescente. 
En otros estudios realizados con adolescentes de entre 1214 años, se ha asociado el comportamiento agresivo del adolescente con un estilo de apego inseguro evitativo o ansioso y se confirma una relación significativa entre dichas variables (Brando, Valera y Zarate, 2008). Del mismo modo, se ha demostrado que un apego seguro facilita el bienestar y el ajuste social del adolecente, además de ser un factor protector de los comportamientos problemáticos (Montañés et al., 2008).

Por otro lado, también el clima familiar puede ser otro factor favorecedor o preventivo de las conductas agresivas en la adolescencia, con correlaciones significativas entre ambas variables (Matalinares et al., 2010). En este sentido, se ha observado que este aspecto puede cumplir un papel significativo para establecer un estado de bienestar resultante de las relaciones que se dan entre los miembros de la familia, reflejado por el grado de comunicación, cohesión e interacción, así como el nivel de organización familiar y el control que ejercen sus miembros.

Del mismo modo que la falta de relaciones positivas y significativas en la familia pueden provocar un aumento de conductas desadaptativas y agresivas, la presencia de una base significativa y sólida en la familia marcada por la percepción subjetiva de satisfacción, puede implicar el desarrollo de conductas adaptativas y un mejor manejo de las emociones y de la IE.

Por ello, es posible que una percepción de insatisfacción familiar facilite la aparición de una baja IE en la adolescencia (Ticona y Zubieta, 2010) y a la inversa, una elevada satisfacción familiar puede facilitar la aparición de una elevada IE, siendo la satisfacción o insatisfacción el elemento principal al ser un indicador de la cohesión y adaptabilidad que maneja el grupo familiar. Según esta premisa, la satisfacción familiar estable, aseguraría unos mejores vínculos entre los miembros del grupo familiar; además, indicaría que existe cierto grado de vinculación emocional positiva entre ellos, aceptándose y confiando los unos en los otros (Bernal, 2008).

Diversos estudios dan muestra de una relación significativa entre satisfacción familiar e IE, en relación al funcionamiento familiar (cohesión y adaptación) e IE (Godoy, 2017), entre comunicación familiar e IE (Rodríguez et al., 2018) y entre satisfacción familiar e IE (Novoa, 2019) o muestran una relación significativa de la influencia de la familia sobre el autoconcepto y la empatía del adolescente, como componentes esenciales de la IE (Segunda, 2016). Si analizamos los estudios realizados con población adolescente, Novoa (2019) realizó un estudio en Perú en el que halló relación estadísticamente significativa y directa entre la satisfacción familiar y la IE, concluyendo que a mayor nivel de satisfacción familiar habrá un mayor nivel de IE en estudiantes de secundaria. En otro estudio llevado a cabo por Segunda (2016) se analiza la influencia de la familia sobre el autoconcepto y la empatía de los adolescentes $(N=$ 332) y se observa una relación directa de los modelos parentales y el clima social familiar en el autoconcepto y la empatía de los hijos adolescentes de entre 12 y 18 años. Dicho autor concluye que existen distintos tipos de dimensiones en los modelos parentales que influyen en el autoconcepto y en la empatía de los adolescentes.

Estudios más recientes, relacionan de forma estrecha y directa el bienestar subjetivo de jóvenes adultos de 18-24 años $(N=439)$ en relación a la IE y la flexibilidad cognitiva y los estilos parentales que se dan en la familia, resaltando el estilo democrático, cálido y la responsabilidad parental como aspectos favorecedores de la flexibilidad cognitiva, la inteligencia emocional y el bienestar subjetivo de los mismos (Wu, Chen y Jen, 2020). Así como Szcześniak y Tułecka (2020), observaron que el funcionamiento familiar y la satisfacción familiar están influidas por el rol moderador que cumple la IE. En su estudio, realizado con 204 adultos de entre 18 y 70 años, se observó que tanto la satisfacción con la vida y la IE correlacionaban de forma positiva y significativa con la cohesión, flexibilidad, comunicación y satisfacción familiar. Especialmente, la IE, influía parcialmente en seis dimensiones del funcionamiento familiar (cohesión, flexibilidad, comunicación, satisfacción familiar, desacuerdo y caos) y en la satisfacción familiar. Estas cuestiones implicaban que aquellos individuos que evalúan su familia como cohesionada, flexible, comunicativa y completa, son más propensos a procesar mejor sus emociones y a disfrutar más de la vida, frente a aquellos que presentan desencuentros y caos, que pueden presentar menos habilidad en el manejo de sus propias emociones y una menor satisfacción vital.

\section{Metodologia}

\section{Muestra}

La muestra estuvo compuesta por 190 estudiantes de dos institutos de educación secundaria de Zaragoza y provincia. Los datos se obtuvieron en un periodo de dos semanas. La edad mínima fue de 15 años y la máxima de $18(M=15,79$; $D T=0,983)$ (ver tabla 1). Los participantes que contestaron al cuestionario pertenecían a los cursos de $1^{\circ}$ a $4^{\circ}$ de Educación Secundaria Obligatoria (ESO). El pase de pruebas se realizó por dos investigadores en los propios centros de formación. 
Tabla 1

Análisis descriptivo de la muestra

\begin{tabular}{cccc}
\hline Edad Mínimo & Máximo & Media & Desv. Típica \\
\hline 15 & 18 & 15,79 & 0,983 \\
\hline
\end{tabular}

\section{Frecuencias Porcentajes}

\begin{tabular}{lll}
15 & 100 & $52,6 \%$ \\
16 & 44 & $23,2 \%$ \\
17 & 31 & $13,6 \%$ \\
18 & 15 & $7,9 \%$ \\
\hline
\end{tabular}

\section{Procedimiento}

El estudio llevado a cabo es un estudio de carácter descriptivo, transversal simple y ex post facto. Los estudiantes recibieron instrucciones concretas de cómo contestar a los cuestionarios por parte de los psicólogos encargados de pasar las pruebas. La duración de ambas pruebas fue de 60 minutos. Una vez terminada, se recogieron los ejemplares y se corrigieron, a continuación, se introdujeron los resultados en una base de datos. Para el análisis estadístico se utilizó el programa SSPS-24. Los estadísticos utilizados han sido pruebas descriptivas, una correlación de Pearson, un Análisis de Varianza y un análisis de clúster.

\section{Instrumentos}

Se utilizaron el STAXI de agresividad, el TMMS-24 para medir la inteligencia Emocional, y el ESFA de Satisfacción Familiar.

El STAXI -2 es un cuestionario que describe los estados de ira y sus efectos en la salud mental y física (Miguel et al., 2009). Tiene dos objetivos: por una parte, determinar los componentes de la ira para realizar una evaluación precisa de la personalidad normal y anormal y por otra, proporcio-

Tabla 2.

Correlaciones entre IE, Agresividad y Satisfacción familiar. nar un instrumento para medir las contribuciones de los diversos componentes de la ira en la evolución de determinados problemas de salud. Analiza los diferentes componentes de la ira: Experiencia, Expresión y Control, así como de sus facetas como Estado y como Rasgo a través de 49 eTementos organizados en 6 escalas y 5 subescalas. Permite obtener un índice de cada escala y subescala y un índice general del test.

La TMMS-24 es una versión reducida del TMMS-48 realizado por el grupo de investigación de Fernández-Berrocal, Extremera y Ramos (2004). Está compuesta por las tres dimensiones de la escala original: Atención, Claridad y Reparación. Consta de veinticuatro ítems y se contesta en una escala de cinco niveles. Una cuestión muy importante a la hora de contestar el cuestionario por parte del individuo, es que este lo haga de forma sincera y ágil.

Por último, se utilizó la prueba ESFA (Barraca y López, 2010), para medir la satisfacción con la familia por parte del sujeto. Este cuestionario consta de 27 ítems y trata de evocar preferentemente una respuesta de tipo afectivo. $\mathrm{Su}$ finalidad es la evaluación de la satisfacción familiar expresada por los sujetos a través de distintos adjetivos. El tiempo requerido es de unos diez minutos.

\section{Objetivos}

Conocer la relación entre la satisfacción familiar, la inteligencia emocional y la agresividad en adolescentes.

Identificar diferentes grupos o perfiles personales en función de la satisfacción familiar, la inteligencia emocional y la agresividad en adolescentes.

\section{Resultados}

Según se muestra en los resultados obtenidos en la correlación de Pearson entre las variables del cuestionario de Inteligencia Emocional, TMMS-24, y el cuestionario que evalúa la Ira de los participantes, STAXI, se puede observar una correlación entre la variable Atención en las emociones con la expresión interna de la ira $\left(0,211^{* *}\right)$. Más destacable es la correlación existente entre regulación emocional y las variables del STAXI, así se observa como correlaciona con ira estado $\left(-0,255^{*}\right)$, con control externo $\left(0,296^{* *}\right)$ pero, sobre todo, destaca la correlación con control interno $(0,428 * *)$ y con control total $(0,422 * *)$ (Tabla 2$)$.

\begin{tabular}{|c|c|c|c|c|c|c|c|c|c|c|c|c|}
\hline & Edad & Atención & Claridad & Reparación & $\begin{array}{c}\text { Ira } \\
\text { estado }\end{array}$ & Ira rasgo & $\begin{array}{c}\text { Exp. } \\
\text { Externo }\end{array}$ & $\begin{array}{c}\text { Exp. } \\
\text { Interna }\end{array}$ & $\begin{array}{l}\text { Contro1 } \\
\text { Externo }\end{array}$ & $\begin{array}{l}\text { Control } \\
\text { Interno }\end{array}$ & $\begin{array}{c}\text { Control } \\
\text { Total }\end{array}$ & ESFA \\
\hline Edad & 1 & & & & & & & & & & & \\
\hline Atención & $-0,024$ & 1 & & & & & & & & & & \\
\hline Claridad & 0,032 & 0,141 & 1 & & & & & & & & & \\
\hline Reparación & 0,083 & 0,053 & $0,288 * *$ & 1 & & & & & & & & \\
\hline Ira estado & $-0,086$ & $-0,023$ & $-0,111$ & $-0,225^{* *}$ & 1 & & & & & & & \\
\hline Ira rasgo & 0,035 & 0,093 & $-0,026$ & $-0,109$ & $0,376 * *$ & 1 & & & & & & \\
\hline $\begin{array}{l}\text { Exp } \\
\text { Externo }\end{array}$ & $-0,05$ & 0,101 & 0,104 & $-0,091$ & $0,195 * *$ & $0,462 * *$ & 1 & & & & & \\
\hline $\begin{array}{l}\text { Exp. } \\
\text { Interna }\end{array}$ & 0,053 & $0,211^{* *}$ & $-0,159^{*}$ & $-0,041$ & 0,132 & $0,158^{8}$ & 0,016 & 1 & & & & \\
\hline $\begin{array}{l}\text { Control } \\
\text { Externo }\end{array}$ & $0,155^{*}$ & 0,025 & 0,134 & $0,296^{* *}$ & $-0,214 * *$ & $-0,285^{* *}$ & $-0,278 * *$ & 0,086 & 1 & & & \\
\hline $\begin{array}{l}\text { Control } \\
\text { Interno }\end{array}$ & 0,1 & $-0,083$ & 0,098 & $0,428^{* *}$ & $-0,217^{* *}$ & $-0,258 * *$ & $-0,210 * *$ & 0,034 & $0,485^{* *}$ & 1 & & \\
\hline $\begin{array}{l}\text { Control } \\
\text { Total }\end{array}$ & $0,148^{*}$ & $-0,035$ & 0,134 & $0,422^{* *}$ & $-0,250 * *$ & $-0,315^{* *}$ & $-0,282 * *$ & 0,07 & $0,856^{* *}$ & $0,867^{* 8}$ & 1 & \\
\hline ESFA & $-0,091$ & 0,066 & $0,182^{*}$ & 0,016 & $-0,102$ & $-0,182^{*}$ & $-0,047$ & 0,003 & $-0,008$ & 0,034 & 0,016 & 1 \\
\hline
\end{tabular}


En el Análisis de Varianza entre las diferentes variables de los dos cuestionarios para la realización de un Análisis de Clúster, se observan diferencias significativas en las variables claridad en las emociones $(F=2,777$; Sig. $=0,065)$, Ira rasgo $(F=4,105$; Sig., 0,018$)$, Control Externo $(F=$
4,513; Sig. $=0,012)$, Control Interno $(F=3,105$; Sig. $=$ 0,047), Control total $(F=4,889$; Sig. $=0,009)$ y la escala Satisfacción con la Familia (ESFA) $(F=438.204$; Sig. $=$ 0,000) (Tabla 3).

Tabla 3.

ANOVA de la IE, Agresividad y Satisfacción familiar

\begin{tabular}{ccccccc}
\hline & Clúster & & Error & & F & Sig. \\
\hline & Media cuadrática & gl & Media cuadrática & gl & & \\
\hline Edad & 1,686 & 2 & 0,961 & 187 & 1,755 & 0,176 \\
Atención & 8,073 & 2 & 43,995 & 187 & 0,184 & 0,832 \\
Claridad & 106,135 & 2 & 38,219 & 187 & 2,777 & 0,065 \\
Reparación & 65,237 & 2 & 39,382 & 187 & 1,657 & 0,194 \\
Ira estado & 2,225 & 2 & 3,368 & 187 & 0,661 & 0,518 \\
Ira rasgo & 42,71 & 2 & 10,403 & 187 & 4,105 & 0,018 \\
Exp. Externo & 2,234 & 2 & 3,864 & 187 & 0,578 & 0,562 \\
Exp. Interna & 0,031 & 2 & 3,212 & 187 & 0,01 & 0,99 \\
Control Externo & 21,585 & 2 & 4,783 & 187 & 4,513 & 0,012 \\
Control Interno & 16,171 & 2 & 5,208 & 187 & 3,105 & 0,047 \\
Control Total & 71,696 & 2 & 14,664 & 187 & 4,889 & 0,009 \\
ESFA & 63260,905 & 2 & 144,364 & 187 & 438,204 & 0,000 \\
\hline
\end{tabular}

Posteriormente, se realizó un Análisis de Clúster en donde se establecieron tres grupos. Un primer grupo que comprende a sujetos con puntuaciones bajas en regulación emocional, así como en Ira Rasgo, Control externo y Control total, sin embargo, con puntaciones altas en Satisfacción con la Familia $(N=9)$. Hay un segundo grupo con puntuación más altas en regulación emocional y control total de la Ira y una puntuación media en Satisfacción familiar, que es el grupo más numeroso $(N=129) \mathrm{y}$, por último, un tercer grupo que presenta puntuaciones medias en casi todas las variables de los tres cuestionarios utilizados $(N=52)$ (Tabla 4).

Tabla 4.

Centros de clústeres finales

\begin{tabular}{lrrr}
\hline & 1 & 2 & 3 \\
\hline Edad & 15 & 16 & 16 \\
Atención & 26 & 25 & 25 \\
Claridad & 26 & 25 & 23 \\
Reparación & 24 & 28 & 27 \\
Ira estado & 8 & 9 & 9 \\
Ira rasgo & 12 & 14 & 15 \\
Exp. Externo & 7 & 8 & 8 \\
Exp. Interna & 7 & 7 & 7 \\
Control Externo & 7 & 9 & 8 \\
Control Interno & 8 & 9 & 8 \\
Control Total & 14 & 18 & 16 \\
ESFA & 213 & 139 & 98 \\
Total sujetos & 9 & 129 & 52 \\
\hline
\end{tabular}

\section{Discusión y conclusiones}

Los resultados obtenidos en esta investigación permiten establecer una correlación entre inteligencia emocional e ira, concretamente la dimensión de atención emocional correlaciona con la expresión interna de la ira, es decir, con la frecuencia con la que los sentimientos de la ira son experimentados, pero no expresados. Dichos resultados irían en la línea de los obtenidos por Extremera y Fernández Berrocal (2002), Bohnert et al. (2003), Siu (2009), Garaigordobil y Oñederra (2010) y Cerezo, Carpio, García y Casanova (2016), que asocian bajas puntuaciones de inteligencia emocional con agresividad conductual en adolescentes. Inglés et al. (2014), encuentran que los sujetos que presentan más comportamientos agresivos, tienen mayor dificultad para controlar la ira y comprender las emociones.

Por otra parte, en esta investigación también se ha hallado que la regulación de las emociones de los adolescentes, correlaciona positivamente con el control de los propios sentimientos y la expresión de la ira. Dicho resultado coincidiría con los estudios de Ciarrochi et al. (2001) y León (2009) que asocian un escaso control de las propias emociones con interacciones positivas inadecuadas, aumento de conflictos interpersonales y aparición de problemas conductuales y conductas disruptivas. A su vez, Inglés et al. (2014) asocian una alta inteligencia emocional rasgo con bajas puntuaciones en conducta agresiva. Diosa et al. (2013) también encuentran que la agresividad está asociada 
con un déficit de la comprensión y la regulación emocional. Cerezo et al. (2016) relacionan negativamente las dimensiones de claridad y reparación emocional con todas las medidas de agresividad. Trinidad y Johnson (2002) indican que los adolescentes que tienen un mayor control de sus emociones, afrontan mejor los conflictos interpersonales. Mestre et al. (2012) indican que el desarrollo de la empatía implicada en la disposición social, contribuye a una conducta prosocial contrapuesta a la conducta agresiva. Finalmente, López del Pino et al. (2008), señalan la impulsividad como la principal predictora de la conducta agresiva.

En este estudio se han establecido tres grupos de sujetos diferenciados principalmente por las puntuaciones obtenidas en la escala ESFA. Ruiz y Carranza (2018) encuentran una correlación directa entre el clima familiar y la inteligencia emocional. En la misma línea los resultados obtenidos por Paéz, Campos, Zubieta et al. (2006) asocian una relación más cálida y una expresividad emocional mayor de los progenitores con una mayor inteligencia emocional. De la Torre-Cruz et al. (2014) también indican que existe una asociación entre un estilo parental autoritario y manifestaciones de agresividad. Un estilo de apego inseguro evitativo o ansioso también incrementa los comportamientos agresivos (Brando et al., 2008). Domenech (2017) concluye en su estudio la educación familiar basada en el apoyo, la comunicación y los vínculos de apego, facilita la conducta prosocial, la aceptación de iguales y las estrategias de afrontamiento funcionales, centradas en el problema. En cambio la crianza basada en la autonomía extrema potencia conductas inadaptadas, como la agresividad, la inestabilidad emocional y estrategias de afrontamiento disfuncionales, centradas en la emoción.

Segunda (2016), Godoy (2017), Rodríguez et al. (2018), Novoa (2019), Wu et al. (2020) y Szczesniak y Tulecha (2020), establecen en sus distintas investigaciones que aquellos adolescentes que perciben unas relaciones satisfactorias con su familia, presentan una mayor inteligencia emocional y una menor manifestación de comportamientos agresivos.

Aunque sí que las investigaciones citadas establecen una clara relación entre IE y agresividad, los resultados son dispares a la hora de profundizar en cada una de las dimensiones de ambas variables. Lo que sí que parece claro, tal y como se ha argumentado a lo largo de este trabajo, es que la presencia de una base familiar sólida, unida a la percepción subjetiva de la satisfacción hacia la familia, favorece una gestión emocional más saludable y por consiguiente, unos comportamientos más adaptativos. Los individuos que afirman pertenecer a familias cohesionadas, flexibles y comunicativas, disfrutan más de la vida porque afrontan las situaciones intrapersonales e interpersonales con estilos positivos y saludables. Por lo que cabría realizar más estudios en esta línea para profundizar en dichas relaciones.

\section{Referencias}

Bar-On, R. (2000). Emotional and social intelligence: insights from the Emotional Quotient Inventory. En Bar-On, R. \& Parker J. (Eds.). The Handbook of Emotional Intelligence. San Francisco: Jossey-Bass.

Bernal, T. (2008). La mediación, una solución a los conflictos de ruptura de pareja. Madrid: Colex.

Brando, M., Valera, J.M. y Zarate, Y. (2008). Estilos de apego y agresividad en adolescentes. Segunda Eрoca, 27(1), 16-42.

Buss, A.H. (1961). The psychology of agression. New York: Wiley.

Buss, A.H. y Perry, M. (1992). The aggression questionnaire. Journal Personality of Social Psychology, 63 (3), 452-459. https://doi.org/10.1037/00223514.63.3.452

Bohnert, A.M., Crnic, K.A. y Lim, K.G. (2003). Emotional competence and aggressive behavior in school-age children. Journal of Abnormal Child Psychology, 31 (1), 79-91. https://doi.org/10.1023/ a:1021725400321.

Carrasco, M.A. y González, M.J. (2006). Aspectos conceptuales de la agresión: definición y modelos explicativos. Acción Psicológica, 2(4), 7-38.

Cerezo, M.T., Carpio, M., García, M. y Casanova, P.F. (2016). Relaciones entre inteligencia emocional, agresividad y satisfacción vital en universitarios. En J.L. Castejón (Coord.). Psicología y Educación: presente y futuro (pp. 1312-1319). ACIPE. http:// hdl.handle.net/10045/63969.

Ciarrochi, J., Chan, A. y Bajgar, J. (2001). Measuring emotional intelligence in adolescents. Personality and Individual Differences, 31(7): 1105-1119. https:// doi.org/10.1016/S0191-8869(00)00207-5

Contini, E.N., Cohen, S., Coronel, C.P. y Mejail, S. (2012). Agresividad y retraimiento en adolescentes. Ciencias Psicológicas, 6(1): 17-28. https://doi.org/ 10.22235/cp.v6i1.59

Damásio, A. (2005). En busca de Spinoza: neurobiología de la emoción y los sentimientos. Madrid: Crítica.

De la Torre-Cruz, M., García-Linares, M.C. y CasanovaArias, P.F. (2014). Relaciones entre estilos educativos parentales y agresividad en adolescentes. Electronic Journal of Research in Educational Psychology, 12(1), 147-170. DOI: https://doi.org/ dx.doi.org/10.14204/ejrep.32.13118

Diosa, J.F., Rodríguez, M. y Romero, F.A. (2013). Inteligencia emocional en un grupo de adolescentes del grado once de una Institución Educativa del Municipio De Envigado (Antioquia), Colombia: una prueba piloto. Psicoespacios, 7(11), 2145-2776. https:// doi.org/10.25057/21452776.220.

Domenech, A. (2017). Comportamiento y emociones en la infancia media y tardía. Contexto familiar y social. https://doi.org/roderic.uv.es/handle/10550/61256. 
Extremera, N. y Fernández-Berrocal, P. (2002). La evaluación de la inteligencia emocional en el aula como factor protector de diversas conductas problema: violencia, impulsividad y desajuste emocional. En F. A. Muñoz, B. Molina y F. Jiménez (Eds.). Actas del I Congreso Hispanoamericano de Educación y Cultura de Paz (599-605). Granada: Universidad de Granada.

Extremera, N. y Fernández Berrocal, P. (2005). Perceived emotional intelligence and life satisfaction: Predictive and incremental validity using the Trait Metamood scale. Personality and Individual Differences, 39, 937-948. https://doi.org/10.1016/ j.paid.2005.03.012.

Fernández-Berrocal, P., Extremera, N. y Ramos, N. (2004). Validity and reliability of the Spanish modified version of the Trait Meta-Mood Scale. Psychological reports, 94(3), 751-755. https://doi.org/10.2466/ PR0.94.3.751-755

Ferragut, M. y Fierro, A. (2012). Inteligencia emocional, bienestar personal y rendimiento académico en preadolescentes. Revista Latinoamericana de Psicología, 44, 95-104.

Freud, S. [1915] (1976). Pulsiones y destinos de pulsión. Tomo XIV. Buenos Aires: Amorrortu.

Garaigordobil, M. y Oñederra, J.A. (2010). Inteligencia emocional en las víctimas de acoso escolar y en los agresores. European Journal of Education and Psychology, 3(2), 243-256. https://doi.org/10.30552/ ejep.v3i2.55

Gardner, H. (2001). Estructuras de la mente. La Teoría de las Inteligencias Múltiples. México: FCE

Goleman, D. (1996). Inteligencia emocional. Barcelona: Kairós.

Godoy, M. (2017). Funcionamiento familiar e inteligencia emocional en familias numerosas y no numerosas. Madrid: Facultad de Ciencias Humanas y Sociales. Universidad Pontificia de Comillas. Recuperado de https://repositorio.comillas.edu/rest/ bitstreams/116202/retrieve.

Inglés, C.J., Torregosa, M.S., García-Fernández, J.M., Martínez-Monteagudo, M.C., Estévez, E. y Delgado, B. (2014). Conducta agresiva e inteligencia emocional en la adolescencia. European Journal of Education and Psychology, 7(1), 29-41. https:// doi.org/10.30552/ejep.v7i1.97

Kohn, M. (1977). Social competence, symptoms and underachievement in childhood. A longitudinal perspective. Nueva York: Holt, Rinchart and Winston

Laplanche, J; Pontalis, J. y Lagache, D. (Dir.) (2004). Diccionario de psicoanálisis. Buenos Aires: Paidós.

León, B. (2009). Salud mental en las aulas. Revista de Estudios de Juventud, 84, 66-83.

López del Pino, C., Sánchez, A., Pérez, M.A. y Fernández, M.P. (2008). Impulsividad, autoestima y control cognitivo en la agresividad del adolescente. EduPsykhé, 7(1), 81-99.

Matalinares, M., Arenas, C., Sotelo, L., Díaz, G., Dioses, A., Yaringaño, J., Muratta, R., Pareja, C. y Tipacti, T. (2010). Clima familiar y agresividad en estudiantes de secundaria de Lima Metropolitana. Revista IIPSI, 13(1), 109-128. https://doi.org/10.15381/ rinvp.v13i1.3740.

Mayer, J.D. y Salovey, P. (1997). What is emotional intelligence? En P. Salovey y D. Sluyter (Coord.). Emotional development and emotional intelligence: Implications for educators. New York: Basic Books.

Mestre, V., Samper, P., Tur-Porcar, A.M., Richaud de Minzi, M.C. y Mesurado, B. (2012). Emociones, estilos de afrontamiento y agresividad en la adolescencia. Universitas Psychologica, 11(4), 1275. https:// doi.org/10.11144/Javeriana.upsy11-4.eeaa

Miguel, J.J., Casado, M.I, Cano, A. y Spielberger, C.D. (2009). STAXI-2. Inventario de expresión de ira estado-rasgo. Madrid: TEA.

Montañés, M., Bartolomé, R., Montañés, J. y Parra, M. (2008). Influencia del contexto familiar en las conductas adolescentes. Ensayos, 17, 391-407.

Novoa, M. (2019). Satisfacción familiar e inteligencia emocional en estudiantes de nivel secundaria del Distrito de Villa María del Triunfo. Tesis de Licenciatura. Lima: Universidad Autónoma del Perú.

Oliva, A. (2006). Relaciones familiares y desarrollo adolescente. Anuario de psicología, 37(3), 209-223.

Páez, D., Campos, M., Zubieta, E. y Casullo, M. (2006). Apego seguro, vínculos parentales, clima familiar e inteligencia emocional: socialización, regulación y bienestar. Ansiedad y Estrés, 12, 329-341.

Real Academia Española (2020). Diccionario de la lengua española [Dictionary of the Spanish Language] (23rd ed.). Madrid, Spain.

Rodríguez, H., Luján, I., Díaz, C., Rodríguez, J. y González, Y. (2018). Satisfacción familiar, comunicación e inteligencia emocional. International Journal of Developmental and Educational Psychology, 1(1), 117-128. https://doi.org/10.17060/ ijodaep.2018.n1.v1.1171.

Ruiz, P. y Carranza, R.F. (2018). Inteligencia emocional, género y clima familiar en adolescentes peruanos. Acta Colombiana de Psicología, 21(2), 188-199. https://www.dx.doi.org/10.14718/ACP.2018.21.2.9

Segunda, M. (2016). Influencia de la familia en el autoconcepto y la empatía de los adolescentes. Tesis doctoral. Universidad de Valencia: Valencia.

Silva, F, Martínez, R. y Generós, G. (1997). La evaluación de la orientación interpersonal: revisión de una línea de investigación. Rev. de Psicología General y Aplicada, 50(1): 85-112.

Siu, A.F.Y. (2009). Trait emotional intelligence and its 
relationships with problem behavior in Hong Kong adolescents. Personality and Individual Differences, 47, 553-557. https//doi.org/10.1016/ j.paid.2009.05.004.

Steinberg, L. (2001). We know some things: Parentadolescent relationships in retrospect and prospect. Journal of Research on Adolescence, 11, 1-19. https://doi.org/10.1111/1532- 7795.00001

Sternberg, R.J. (1986). Las Capacidades Humanas: Un Enfoque Desde el Procesamiento de la Información. Labor.

Szcześniak, M. y Tułecka, M. (2020). Family Functioning and Life Satisfaction: The Mediatory Role of Emotional Intelligence. Psychology Research and Behavior Managment, 13, 223-232. https:// doi.org/10.2147/PRBM.S240898.

Ticona, M. y Zubieta, R. (2010). Satisfacción familiar e inteligencia emocional en estudiantes de la I.E. Héroes del Cenepa (Tesis de pregrado). Universidad Nacional de San Agustín. Arequipa: Perú.

Trinidad, D. y Johnson, C. (2002). The association between emotional intelligence and early adolescent tobacco and alcohol use. Personality \& Individual Differences, 32. https://doi.org/10.1016/S0191-8869(01) 00008-3.

Wu, C., Chen, W. y Jen, C. (2020). Emotional Intelligence and Cognitive Flexibility in the Relationship Between Parenting and Subjective Well-Being, Journal of Adult Development. https://doi.org/10.1007/ s10804-020-09357-x 